\title{
Magnetic field amplification at planetary and astrophysical high Mach-number shocks
}

\author{
Artem Bohdan, ${ }^{a, *}$ Martin Pohl, ${ }^{a, b}$ Jacek Niemiec, ${ }^{c}$ Paul J. Morris, ${ }^{a}$ Yosuke \\ Matsumoto, ${ }^{d}$ Takanobu Amano, ${ }^{e}$ Masahiro Hoshino ${ }^{e}$ and Ali Sulaiman ${ }^{f}$ \\ ${ }^{a}$ DESY, DE-15738 Zeuthen, Germany \\ ${ }^{b}$ Institute of Physics and Astronomy, University of Potsdam, DE-14476 Potsdam, Germany \\ ${ }^{c}$ Institute of Nuclear Physics Polish Academy of Sciences, PL-31342 Krakow, Poland \\ ${ }^{d}$ Department of Physics, Chiba University, 1-33 Yayoi-cho, Inage-ku, Chiba 263-8522, Japan \\ ${ }^{e}$ Department of Earth and Planetary Science, the University of Tokyo, 7-3-1 Hongo, Bunkyo-ku, Tokyo \\ 113-0033, Japan \\ ${ }^{f}$ Department of Physics and Astronomy, University of Iowa, IA, USA \\ E-mail: artem.bohdan@desy.de
}

Collisionless shocks are present everywhere in the universe, from the solar environment to distant supernovae. They are often associated with strong magnetic fields due to strong nonthermal radiation. However, it is still not well understood how magnetic fields are amplified at scales of the shock thickness. Here we use a set of large-scale Particle-In-Cell (PIC) simulations of non-relativistic perpendicular shocks in the high Mach number regime to clarify this issue. These shocks are Weibel-instability mediated. We present the evidence that the magnetic field is amplified in the shock transition due to the Weibel instability and the magnetic field strength strongly correlates with the Alfvénic Mach number. We propose a new explanation for this correlation. PIC simulation results can explain in-situ magnetic field measurements of Saturn's bow shock performed by the Cassini spacecraft.

$37^{\text {th }}$ International Cosmic Ray Conference (ICRC 2021)

July 12 th - 23rd, 2021

Online - Berlin, Germany

\footnotetext{
*Presenter
} 


\section{Introduction}

Collisionless shocks are known as efficient particle accelerators. They can be found in planetary systems, supernova remnants (SNRs), jets of active galactic nuclei, galaxy clusters, etc. These shocks are often magnetized, and magnetic fields play a key role in their physics. Indeed, the jump conditions for the magnetic field, the internal shock structure and dynamics, and particle acceleration mechanisms strongly depend on the magnetic field strength and structure in the shock vicinity.

Nonrelativistic high-Mach-number SNR shocks are of particular interest due to their ability to produce the most part of galactic cosmic rays. Amplified magnetic fields in the shock vicinity have been inferred from observations of SNRs through the detection of non-thermal X-ray rims [1], fast temporal variability of X-ray hot spots [2], and the $\gamma$-ray/X-ray flux ratio [3]. There are various possible mechanisms for magnetic field amplification at the shock: cosmic-ray driven nonresonant modes [4], fluid vorticity downstream of the shock seeded by upstream density inhomogeneities [5], cosmic ray pressure driven magnetic field amplification [6], etc. However, all these processes operate on scales larger than the upstream ion gyroradius.

Magnetic field amplification mechanisms on scales smaller than the upstream ion gyroradius (or the shock width) at high-Mach-number quasi-perpendicular shocks are still not well understood. Radio and X-ray observations of synchrotron radiation from SNRs emitted by relativistic electrons cannot reveal magnetic-field properties at such small scales. However, in the Solar system there is another example of a well studied high-Mach-number shock, namely the bow shock of Saturn's magnetosphere. In-situ measurements by the Cassini spacecraft [7] have revealed the detailed magnetic field structure of Saturn's bow shock with resolution below the ion gyroradius. Note, that the Alfvénic Mach number of this shock can reach values around 200 which is similar to that of SNR shocks. A strong positive correlation between the normalized overshoot magnetic-field strength and the Alfvénic Mach number of the shock is observed at the Saturn's bow shock across the entire range of measured $M_{A}$. The ratio of the maximum and the upstream field strength reaches 50 .

Leroy's calculations [8] for perpendicular high Mach number shocks combined with hybrid simulations suggest that the overshoot magnetic-field strength $\left(B_{\text {over }}\right)$ can be estimated as:

$$
B_{\text {over }} \approx 0.4 B_{0} M_{\mathrm{A}}^{7 / 6}
$$

where $B_{0}$ is the upstream field strength. In this model the magnetic-field amplification is associated only with plasma compression. However, 3D PIC simulations [9], laboratory experiments [10] and in-situ measurements of the Earth's bow shock in the high-Mach number regime [11] demonstrate amplification of the upstream magnetic field due to the ion-ion filamentation/Weibel instability [12], which results from the interaction of the upstream and shock-reflected ions.

Here we discuss a mechanism of magnetic-field amplification that is based on a realistic description of perpendicular nonrelativistic high-Mach-number shocks and can explain the correlation between the field strength and the Alfvénic Mach numbers observed with Cassini at Saturn's bow shock. 


\begin{tabular}{lcccccccccc}
\hline \hline Runs & $m_{\mathrm{i}} / m_{\mathrm{e}}$ & $M_{\mathrm{A}}$ & \multicolumn{2}{c}{$M_{\mathrm{s}}$} & \multicolumn{2}{c}{$\beta_{\mathrm{e}}$} & \multicolumn{3}{c}{$\left|B_{\mathrm{sh}}\right| / B_{0}$} & \multicolumn{2}{c}{$U_{\mathrm{sh}, \mathrm{B}} / U_{\mathrm{sh}, \mathrm{i}}$} \\
& & & ${ }^{*} 1$ & ${ }^{*} 2$ & ${ }^{*} 1$ & $*_{2}$ & $* 1$ & ${ }^{*} 2$ & ${ }^{*}$ & ${ }^{*} 2$ \\
\hline \hline $\mathrm{A} 1, \mathrm{~A} 2$ & 50 & 22.6 & 1104 & 35 & $5 \cdot 10^{-4}$ & 0.5 & 0.17 & 0.17 & 9.4 & 9.3 \\
$\mathrm{~B} 1, \mathrm{~B} 2$ & 100 & 31.8 & 1550 & 49 & $5 \cdot 10^{-4}$ & 0.5 & 0.15 & 0.13 & 12.4 & 11.6 \\
$\mathrm{C} 1, \mathrm{C} 2$ & 100 & 46 & 2242 & 71 & $5 \cdot 10^{-4}$ & 0.5 & 0.12 & 0.11 & 16.1 & 15 \\
$\mathrm{D} 1, \mathrm{D} 2$ & 200 & 32 & 1550 & 49 & $5 \cdot 10^{-4}$ & 0.5 & 0.17 & 0.16 & 13.4 & 12.9 \\
$\mathrm{E} 1, \mathrm{E} 2$ & 200 & 44.9 & 2191 & 69 & $5 \cdot 10^{-4}$ & 0.5 & 0.12 & 0.11 & 15.5 & 14.8 \\
$\mathrm{~F} 1, \mathrm{~F} 2$ & 400 & 68.7 & 3353 & 106 & $5 \cdot 10^{-4}$ & 0.5 & 0.074 & 0.067 & 18.7 & 17.8 \\
$\mathrm{G} 1, \mathrm{G} 2$ & 50 & 68.7 & 3353 & 106 & $5 \cdot 10^{-4}$ & 0.5 & 0.083 & 0.081 & 19.8 & 19.6 \\
$\mathrm{H} 1^{\dagger}, \mathrm{H} 2$ & 50 & 100 & 4870 & 154 & $5 \cdot 10^{-4}$ & 0.5 & - & 0.039 & - & 19.9 \\
$\mathrm{I}^{\dagger}, \mathrm{I} 2$ & 50 & 150 & 7336 & 232 & $5 \cdot 10^{-4}$ & 0.5 & - & 0.024 & - & 23.6 \\
\hline
\end{tabular}

Table 1: Parameters of simulation runs described in this paper. Listed are: the ion-to-electron mass ratio, $m_{\mathrm{i}} / m_{\mathrm{e}}$, the Alfvénic and sonic Mach number, $M_{\mathrm{A}}$ and $M_{\mathrm{s}}$, the electron plasma beta, $\beta_{\mathrm{e}}$, and measured $\left|B_{\mathrm{sh}}\right| / B_{0}$ and $U_{\mathrm{sh}, \mathrm{B}} / U_{\mathrm{sh}, \mathrm{i}}$ at the shock. Some values are shown separately for the left (runs $* 1$ ) and the right (runs $* 2$ ) shock. Results for runs marked with " ${ }^{\prime}$, are not discussed in this letter because of the strong numerical artifacts. All runs use the in-plane magnetic field configuration, $\varphi=0^{\circ}$.

\section{Simulation setup}

We perform shock simulations using an optimized fully-relativistic electromagnetic $2 \mathrm{D}$ code with MPI parallelization developed from TRISTAN $[13,14]$ with an in-plane magnetic-field configuration which permits a good approximation of realistic 3D shocks $[9,15]$. Shocks are initialized with a flow-flow method. The collision of two counterstreaming electron-ion plasma flows spawns two independent shocks propagating in opposite directions. Shock are propagating in media which differ only by the electron plasma beta, the ratio of the electron plasma pressure to the magnetic pressure, which is $5 \cdot 10^{-4}$ and 0.5 for the left and the right shocks, respectively.

The large-scale magnetic field, $\mathbf{B}_{\mathbf{0}}$, is perpendicular to the shock normal $\left(\theta_{B n}=90^{\circ}\right)$ and it lies in the simulation plane (the in-plane configuration, $\varphi=0^{\circ}$ ). Our simulations cover a wide range of physical and numerical parameters: $M_{\mathrm{A}}=22.6-150, m_{\mathrm{i}} / m_{\mathrm{e}}=50-400$ and $\beta_{\mathrm{e}, \mathrm{R}}=5 \cdot 10^{-4}-0.5$. Hence, we can compare our simulation results with data for Saturn's bow shock for $M_{\mathrm{A}} \geqslant 20$.

\section{Magnetic field amplification by the Weibel instability}

Figure 1(a) shows the electron-density map of the fully developed shock from run B2. The shock position, $x_{\mathrm{sh}} \approx 0$, is defined as a location of the shock overshoot. Buneman waves are visible as small-scale density ripples at $x-x_{\mathrm{sh}} \approx(8-12) \lambda_{\mathrm{si}}$. The Weibel instability is visible through the density filaments at $x-x_{\mathrm{sh}} \approx(2-10) \lambda_{\mathrm{si}}$. The downstream is a region behind the overshoot, at $x-x_{\mathrm{sh}} \lesssim-5 \lambda_{\mathrm{si}}$. The described structure is representative for all runs and in general for high-Mach-number shocks [15-21].

Figure 1(b) displays the density and magnetic-field profiles in the shock transition for run B2. The profiles are averaged in time over two cycles of the shock reformation. The plasma compression reaches $N_{\text {over }} / N_{0} \approx 7$. The same plasma compression is observed in all simulations, which is not in 


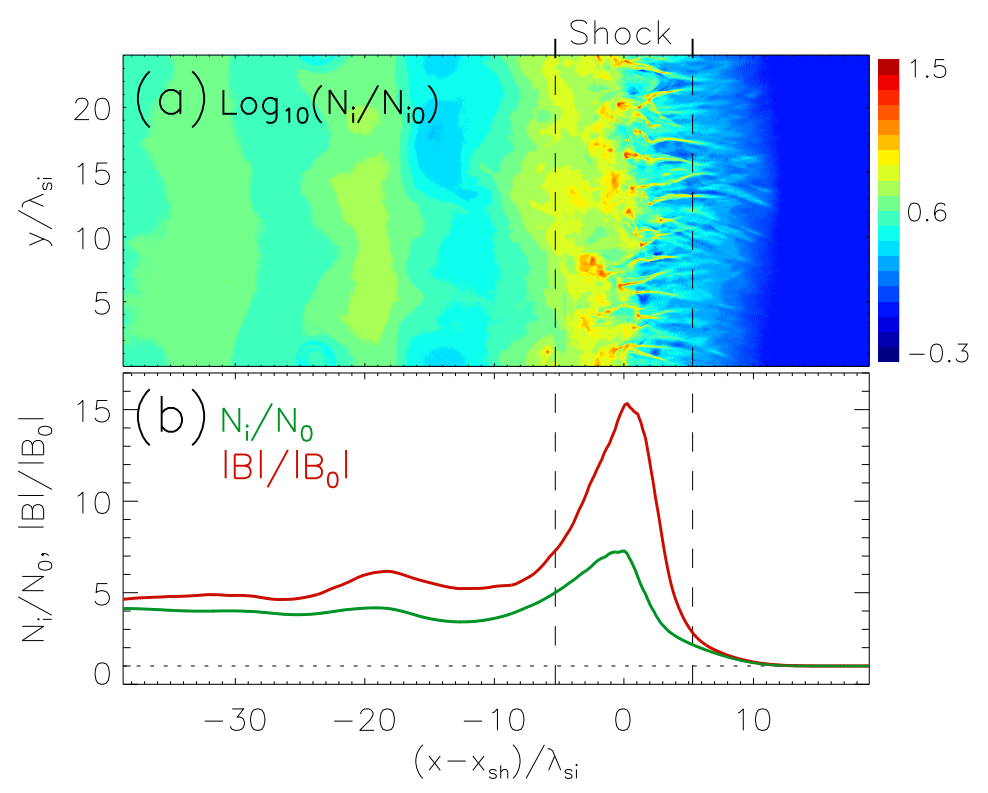

Figure 1: Density and magnetic field for run B2. Panel (a): ion density in logarithmic scale. Panel (b): red line - the profile of normalized ion density, green line - the profile of normalized magnetic field. Profiles are calculated in the shock reference frame and averaged over the shock reformation cycle. The shock region is marked by dashed lines, and $x_{\mathrm{sh}}$ is the shock position.

line with Leroy's model, which predicts $N_{\text {over }} / N_{0} \approx 23$. The field strength in run B2 increases twice as much as does the density, indicating substantial noncompressional magnetic-field amplification.

Strong divergence between density and magnetic-field profiles at the shock transition arises from the growth of $B_{\mathrm{x}}$ and $B_{\mathrm{z}}$ due to folding of the magnetic field by the Weibel modes whose wave vector is perpendicular to the relative velocity of shock-reflected and incoming upstream ions. Further straightening of magnetic-field lines leads to a rapid reduction of magnetic field downstream of the overshoot, which reaches $\left|B_{\text {down }}\right| /\left|B_{0}\right| \approx 4$ within a few ion gyroradii behind the shock.

We define the shock region as a sector of width $L_{\mathrm{sh}}=r_{\mathrm{gi}} / 3$ centered at $x_{\mathrm{sh}}$, where $r_{\mathrm{gi}}=M_{\mathrm{A}} \lambda_{\mathrm{si}}$. The shock region for run B2 is marked with dashed lines in Figure 1. Figure 2 shows the amplitude (panel a) and energy density (panel b) of the magnetic field in the shock region, averaged over the shock self-reformation cycle and with error bars reflecting the level of temporal variation. The normalized field strength, $\left|B_{\mathrm{sh}}\right| / B_{0}$, grows with increasing Alfvénic Mach number and can be estimated as (green line in Fig 2(a)):

$$
\left|B_{\mathrm{sh}}\right| \approx 2 \sqrt{M_{\mathrm{A}}} B_{0}
$$

Therefore the normalized energy density of the magnetic field can be expressed as

$$
\frac{U_{\mathrm{sh}, \mathrm{B}}}{U_{\mathrm{sh}, \mathrm{i}}}=\frac{B_{\mathrm{sh}}^{2}}{\mu_{0} N_{\mathrm{i}} m_{\mathrm{i}} v_{\mathrm{sh}}^{2}} \approx \frac{4}{M_{\mathrm{A}}},
$$

which is a descending trend (green line in Fig 2(b)), that well reproduces the energy density observed in the simulations. Note, that the Weibel instability also transfers energy to electrons via magnetic reconnection [20] at a level that is commensurate with the error bars. 

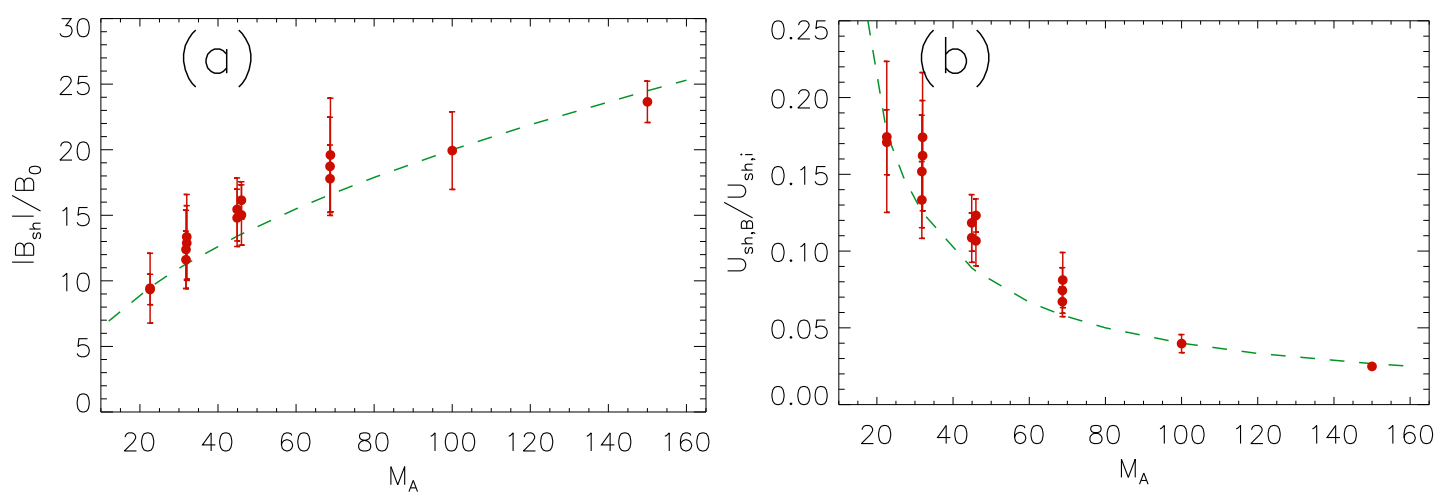

Figure 2: The normalized magnetic-field strength (panel (a)) and the magnetic energy density normalized by the upstream ion energy density (panel (b)), both evaluated in the shock region defined in Fig 1 . The red color corresponds to PIC simulation data. The error bars reflect the temporal variations of the magnetic field due to the shock reformation. The green dotted line in panel (a) reflects $\left|B_{\mathrm{sh}}\right| / B_{0}=2 \sqrt{M_{\mathrm{A}}}$, and that in panel (b) shows $U_{\mathrm{sh}, \mathrm{B}} / U_{\mathrm{sh}, \mathrm{i}}=4 M_{\mathrm{A}}^{-1}$.

The magnetic field downstream of the shock shows the same trends as in the shock region for all runs. It appears that the efficiency of magnetic-field relaxation does not depend on the simulation parameters or the field amplification level. As was mentioned before, the magnetic field remains amplified for only a few ion gyroradii behind the shock, and far downstream the field strength is $4 B_{0}$. Unfortunately, our simulation time is too short to fully capture the entire relaxation especially for high $M_{\mathrm{A}}$, however the data we have suggest that the length scale of the relaxation is roughly proportional to $\left|B_{\text {sh }}\right| /\left|B_{0}\right|$.

Runs that differ only in the mass ratio or the upstream plasma beta show the same results, and we conclude that these parameters do not play a significant role in magnetic-field amplification. Solving the dispersion relation for the Weibel instability [22], we showed that the normalized growth rate of the most unstable mode is proportional to the shock velocity:

$$
\Gamma_{\max } \propto v_{\mathrm{sh}} \omega_{\mathrm{pi}} \quad \text { or } \quad \Gamma_{\max } \propto M_{\mathrm{A}} \Omega_{\mathrm{i}} .
$$

The shock self-reformation limits the development time of the Weibel modes, and the Weibel instability has about $\Omega_{\mathrm{i}}^{-1}$ to develop before a new shock reformation cycle begins. Therefore, Equation 4 shows that the number of exponential growth cycles available for the Weibel modes scales inversely with the Mach number, whatever the shock speed. Hence the Alfvénic Mach number is the only upstream parameter that is relevant for the magnetic-field amplification in the shock transition.

\section{PIC simulations vs in-situ measurements}

Having established that magnetic field amplification exclusively depends on the Alfvénic Mach number, we can directly compare PIC simulation results and in-situ measurements. However, a correction should be made to our formula 2, because the intrinsic shock dynamics also affects the magnetic-field amplification level. It has been shown in [23], that 16 shocks out of 54 shock 


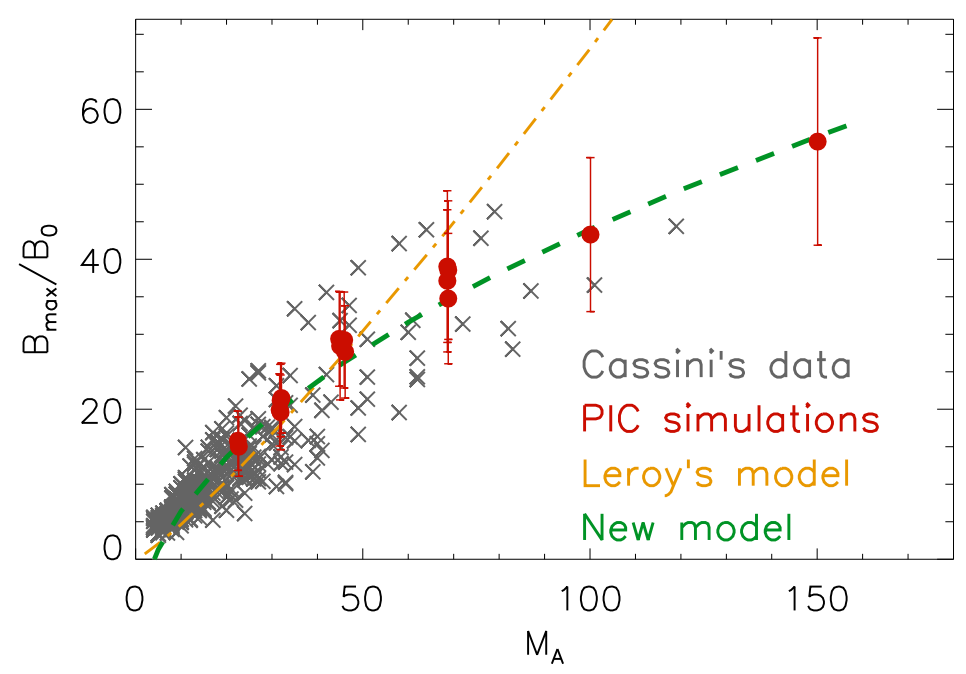

Figure 3: Cassini measurements [7] indicated by gray crosses and PIC simulation data displayed as red dots. The yellow dash-dotted line is an earlier prediction, $B_{\text {over }} / B_{0} \approx 0.4 M_{\mathrm{A}}^{7 / 6} / 1.26$ (cf. Eq. 1). The green dashed line is the behavior found in our PIC simulations, $B_{\max } / B_{0}=5.5\left(\sqrt{M_{\mathrm{A}}}-2\right)$.

crossings undergo shock reformation, and the field amplification at these shocks is 1.42 times that at the other 38 shocks. This behaviour is likely explained by the differences in ion reflection at the shock ramp between reforming and non-reforming shocks. Without cyclic reformation the ion reflection rate is steady and below its maximum value at a reforming shock. The first of two consequences is a stronger magnetic-field amplification in reforming shocks, on account of the proportionality between the peak growth rate of the Weibel modes and the fraction of shockreflected ions. Second, a higher ion-reflection rate implies a higher plasma density in the shock overshoot and a stronger field amplification by compression. Therefore, $B_{\max } / B_{0}$ is higher for shocks at which shock reformation is observed. In all our PIC simulations the shock reformation is clearly visible. To properly compare with the full set of in-situ measurements, we therefore reduce the peak field strength measured in the simulations by a factor of 1.26 , that is derived from the in-situ measurements of [23]. Note that, the Leroy's model also should be corrected by the same factor, because the numerical coefficient in Eq. 1 is deduced from shock simulations where the shock reformation is clearly evident [24].

The largest set of magnetic-field measurements at Saturn's bow shock [7] contains 422 shock crossings during which the shock was quasi-perpendicular, $\theta_{B_{n}} \geqslant 45^{\circ}$, and for which $B_{\max } / B_{0}$ is indicated by gray crosses in Figure 3. To derive $B_{\max } / B_{0}$ from PIC simulation data we mock the straight trajectory of a virtual spacecraft, and then we average $B_{\max } / B_{0}$ over all possible starting points and the speed and flight direction of the imagined spacecraft. The results are shown in Figure 3 as red dots with error bars.

Figure 3 demonstrates a good match between in-situ measurements and the simulation data. A fit of the simulation data is shown as green dashed line in Fig. 3, using a formula

$$
\frac{B_{\max }}{B_{0}}=5.5\left(\sqrt{M_{\mathrm{A}}}-2\right) \text {. }
$$

For comparison, the yellow dash-dotted line in Figure 3 shows the corrected scaling in Eq. 1. 
Although Eq. 1 matches the data reasonably well for $M_{\mathrm{A}}<60$, even that may be a coincidence because this model relies on simplified 1D shock physics. In our view, equation 5 is a better and physically motivated approximation for $B_{\max } / B_{0}$ at shocks with $M_{\mathrm{A}} \gtrsim 10$. This is not a proof that magnetic fields are defined by the Weibel instability at $10<M_{\mathrm{A}}<20$, but at least equation 5 can be used to estimate the field strength.

In addition to the magnetic-field amplification, other Cassini measurements are also in good agreement with PIC simulations. The shock thickness is about one ion gyroradius, and the period of shock self-reformation is $\sim 1.5 \Omega_{\mathrm{i}}^{-1}$. Furthermore, Cassini data show that the magnetic field downstream of the shock quickly stabilizes at the strength predicted by the jump condition indicating the operation of similar mechanisms of magnetic-field amplification and relaxation.

\section{Conclusions}

We have established a strong connection between the Weibel instability and magnetic-field amplification at high-Mach-number shocks due to nonlinear development of the Weibel instability at the shock ramp. In-situ measurements of Saturn's bow shock are fully consistent with the results of PIC simulations. As the Alfvénic Mach number is the only relevant parameter, our findings on the field amplification inside the shock transition layer should also apply to SNR shocks. Weibel modes can increase the local synchrotron emissivity by a factor $\left(B_{\mathrm{sh}} / B_{0}\right)^{2}$, which may reach a thousand. Larger enhancements arise in the X-ray band beyond the synchrotron peak frequency, but overall the effect is likely unobservable with current facilities. However, the interaction of the Weibel modes with other amplification processes may introduce significant changes in the shock structure and it should be taken into account in further studies.

Strong magnetic field generated by the Weibel instability affects particle behaviour, decreasing their gyroradius and introducing additional scattering. It may change the efficiency of stochastic shock drift acceleration (SSDA) of electrons [9], which is expected to operate at quasi-perpendicular shocks. Also stronger magnetic field at the shock transition require larger momenta for particle injection into DSA. We estimate particle injection momenta as

$$
p_{\text {inj }} \propto r_{\mathrm{sh}} B_{\mathrm{sh}} \propto \sqrt{M_{\mathrm{A}}},
$$

where $M_{\mathrm{A}}>20$ and the shock is Weibel-mediated. These connections shall be explored in future studies of collisionless high-Mach-number shocks.

\section{Acknowledgments}

M.P. acknowledges support by DFG through grant PO 1508/10-1. The work of J.N. has been supported by Narodowe Centrum Nauki through research project 2019/33/B/ST9/02569. The numerical experiment was done with the 2.399 PFlop Prometheus system at ACC Cyfronet AGH and North-German Supercomputing Alliance (HLRN) under projects bbp00014 and bbp00033. 


\section{References}

[1] Vink, J. \& Laming, J. M. 2003, ApJ, 584, 758.

[2] Uchiyama, Y., Aharonian, F. A., Tanaka, T., et al. 2007, Nature, 449, 576.

[3] Acciari, V. A., Aliu, E., Arlen, T., et al. 2011, ApJL, 730, L20.

[4] Bell, A. R. 2004, MNRAS, 353, 550.

[5] Giacalone, J. \& Jokipii, J. R. 2007, ApJL, 663, L41.

[6] Downes, T. P. 2012, MNRAS, 425, 2277.

[7] Sulaiman, A. H., Masters, A., \& Dougherty, M. K. 2016, JGR (Space Physics), 121, 4425.

[8] Leroy, M. M. 1983, Physics of Fluids, 26, 2742.

[9] Matsumoto, Y., Amano, T., Kato, T. N., et al. PRL, 119, 105101.

[10] Fiuza, F., Swadling, G. F., Grassi, A., et al. 2020, Nature Physics, 16, 916.

[11] Sundberg, T., Burgess, D., Scholer, M., et al. 2017, ApJL, 836, L4.

[12] Weibel, E. S. 1959, PRL, 2, 83.

[13] Buneman, O., Computer Space Plasma Physics: Simulation Techniques and Software Eds.: H. Matsumoto \& Y. Omura, Tokyo: Terra Scientific, 1993

[14] Niemiec, J., Pohl, M., Stroman, T., et al. 2008, ApJ, 684, 1174.

[15] Bohdan, A., Niemiec, J., Kobzar, O., et al. 2017, ApJ, 847, 71.

[16] Kato, T. N. \& Takabe, H. 2010, ApJ, 721, 828.

[17] Matsumoto, Y., Amano, T., Kato, T. N., et al. 2015, Science, 347, 974.

[18] Bohdan, A., Niemiec, J., Pohl, M., et al. 2019, ApJ, 878, 5.

[19] Bohdan, A., Niemiec, J., Pohl, M., et al. 2019, ApJ, 885, 10.

[20] Bohdan, A., Pohl, M., Niemiec, J., et al. 2020, ApJ, 893, 6.

[21] Bohdan, A., Pohl, M., Niemiec, J., et al. 2020, ApJ, 904, 12.

[22] Bohdan, A., Pohl, M., Niemiec, J., et al. 2021, PRL, 126, 095101.

[23] Sulaiman, A. H., Masters, A., Dougherty, M. K., et al. 2015, PRL, 115, 125001.

[24] Matsumoto, Y., Amano, T., \& Hoshino, M. 2012, ApJ, 755, 109. 